Brigitte Ausfeld-Hafter

\title{
Qui bono? - Zu Kendall DE: «National Center for Complementary and Alternative Medicine (NCCAM) Introduction to Acupuncture with Disclaimer». Schweiz Z Ganzheitsmed 2013;25: DOl: 10.1159/000353908
}

Ungezählt sind die E-Mails, die ich jeweils von erbosten Kollegen erhalte, wenn sie sich über Zeitungsartikel von P.U. Unschuld, H. Lehmann oder D.E. Kendall ärgern. Der Grundtenor besteht im Vorschlag, dass ich mich als emeritiertes Mitglied der Kollegialen Instanz für Komplementärmedizin (KIKOM) und ehemals zuständig für die Traditionelle Chinesische Medizin (TCM) mitzuärgern habe und vehemente Gegenargumente zu diesen Artikeln publizieren soll.

Fakt ist, dass die oben genannten Herren in vielen Bereichen ziemlich recht haben. So ist die TCM, wie wir sie heute kennen, eine chinesische Erfindung der 1960er-Jahre. Davor hiess diese Medizin «alte Medizin», und weil «alt» einen misslichen und pejorativen Beiklang hat, wurde dieses Wort unter Maos Ägide durch «traditionell» ersetzt.

Ebenfalls Fakt ist, dass Qi nicht mit «Energie» übersetzt werden kann, obgleich «Energie» in unserem Sprachgebrauch nur schwammig de- finiert werden kann (es sei denn, wir sprechen von der physikalischen Energie).

Fakt ist zudem, dass die diversen Lehren der Akupunkturschulen in Europa nicht ausschliesslich auf den «unmöglichen Ideen» von Soulié de Morant basieren. Dass die «verschrobenen Ansichten» dieses vor 80 Jahren erschienenen Akupunkturkonzeptes die Grundlagen der Schulen Europas seien - wie Herr Kendall impliziert -, ist eine infame und unzumutbare Unterstellung vonseiten des Autors.

Der Artikel von Herrn Kendall ist ein neuerliches Pamphlet, denn die SCHWEIZERISCHE ZeITSCHRIFT FÜR GANZHEITSMEDIZIN brachte schon im Jahr 2011 einen dem jetzigen ähnlichen Beitrag. Solche Pamphlete sind medienwirksam. Schlechte Nachrichten werden in der heutigen Zeit gern gelesen; da viele Unterstellungen jedoch aus einer relativ einseitigen Sicht verfasst sind, können sie nicht fundiert sein. Herr Kendall möchte wohl die Debatte in den USA ein bis- schen einheizen, und das sei ihm unbenommen.

Dieser Artikel ist von der neuartigen - im Moment sehr aktuellen Idee besessen, dass die Akupunktur auf eine westliche physiologische Basis gestellt werden sollte. Und es stellt sich die Frage, wie ein philosophisch erarbeitetes Grundgerüst in ein naturwissenschaftliches überführt werden kann. Und warum sollen diese zwei verschiedenen Denkansätze, nämlich das linear-analytische Denken und das synthetisch-intuitive Denken, nicht neben- und miteinander bestehen können? Der klinische Alltag zeigt nämlich, dass lineares Denken nicht immer zielführend ist und zum Wohl des Patienten auch ein intuitives Arztdenken eine willkommene Ergänzung bedeuten kann.

Dies ist mein Kommentar zum Artikel und damit einer für die erbosten E-Mail-schreibenden Kollegen.

Literatur bei der Verfasserin auf Anfrage.

\section{KARGER}

Fax +497614520714 Information@Karger.com www.karger.com
(๑) 2013 S. Karger GmbH, Freiburg

Accessible online at: www.karger.com/szg
Dr. med. Brigitte Ausfeld-Hafter

Em. Dozentin der Kollegialen Instanz für Komplementärmedizin (KIKOM)

Universität Bern, Inselspital, Imhoof-Pavillon

3010 Bern, Schweiz

brigitte.ausfeld-hafter@kikom.unibe.ch 